\title{
Infrared Vertical-Cavity Surface-Emitting Lasers with a Multi-Oxide Layer Structure: Small Change Big Effect
}

\author{
Ahmad N. AL-Omari
}

\begin{abstract}
Conventional vertical-cavity surface-emitting lasers (VCSELs) structure generally incorporates 1 to 2 oxidation layers for electrical and optical confinement. Incorporating several oxidation layers (> 2) within the VCSEL's distributed Bragg reflector (DBR) structure is a small and simple to do change, but with big effect. VCSELs structure with multi-oxide layers (MOLs) are expected to demonstrate several advantages over conventional structures. This paper mainly discusses the MOL structure effect on frequency modulation bandwidth $\left(f_{-3 \mathrm{~dB}}\right)$ and slightly touches on single-mode and thermal issues. Simulation results indicated $\sim 68 \%$ improvement in $f_{-3 \mathrm{~dB} \max }$, where conventional and MOL VCSELs exhibited $f_{-3 \mathrm{~dB} \max }$ values of $22 \mathrm{GHz}$ and $37 \mathrm{GHz}$, respectively. In addition, MOL VCSELs maintain single-mode operation with a side mode suppression ratio of $>32 \mathrm{~dB}$. On the other hand, MOL structure demonstrated a degraded performance in terms of reduced thermal conductivity values of $0.084 \mathrm{~W} / \mathrm{cm}-\mathrm{K}$ compared to GaAs and AlAs.
\end{abstract}

Keywords - Multi-oxide layer, VCSELs, Parasitic Capacitance, Single-Mode, Thermal Resistance

\section{INTRODUCTION}

$\mathbf{I}_{(\mathrm{N}) \mathrm{sen}}^{\mathrm{n}}$ vertical-cavity surface-emitting lasers (VCSELs) have emerged as the reliable light sources of choice for several optoelectronic systems and applications due to their small size, planar fabrication of high-density two dimensional arrays, low power consumption, circular optical beam profile necessary for efficient beam coupling, high performance, and simple integration [1]-[3].

VCSELs with high frequency modulation bandwidth are significant components for high-data rate transmission applications. Addressing the current VCSEL's performance limiting factors will extend its potential applications into future high-power applications such as solid state or fiber laser pumping and free-space optical communication [4], high data rate transmission systems, supercomputer interfaces, and multi-channel computing systems [5].

Beside electrical parasitic circuit effects such as : parasitic top and bottom mirrors resistances and parasitic junction, oxide, and pad capacitances [6]-[10], and thermal effects due to self-heating [8], [10]-13], optical beam profile

Electronic Engineering Department, Hijjawi Faculty for Engineering Technology, Yarmouk University, Irbid 21163, Jordan (e-mail: oahmad@yu.edu.jo). On sabbatical leave: Engineering Technology Program, Community College of Qatar, C-Ring, Doha, Qatar (e-mail: ahmad.alomari@ccq.edu.qa), Tel.: +97450019014. characteristics including multi-mode operation [10], [14], [15] can also limit the VCSEL's performance and thus limits the utilization of VCSELs in several potential applications such as high-speed laser printing and engraving, bar code scanning, and single-mode optical fiber data communication.

Although the emitted output optical power can be increased by increasing the oxide aperture diameter (i.e. active area) of a VCSEL, this approach stimulates higher order modes where oxide aperture diameters of less than $4 \mu \mathrm{m}$ are required for VCSELs to emit single transverse mode beams [16]. Additionally, increasing the device's oxide aperture diameter reduces the frequency modulation bandwidth that is inversely proportional to the square root of the effective volume [17]. Alternatively, decreasing the VCSEL's cavity volume which is expected to promote single transverse mode operation and high-speed performance impedes obtaining devices with high output optical power due to the reduced cavity volume size. Thus, realizing VCSELs with improved performance that promotes simultaneously high-speed operation with high output optical power is a challenging issue.

Although several factors such as spatial hole-burning, current uniformity, gain-mode overlap, and optical loss of higherorder modes may contribute to single-mode performance, the size of the oxide aperture diameter (active diameter) is considered one of the critical VCSEL fabrication parameters that can promote signal transverse mode operation. Thus, fabricating VCSELs with small oxide aperture diameters promote high-speed operation and single-mode performance. On the other hand, VCSELs with small oxide apertures exhibit few drawbacks such as the need for crucial alignments during the multi-steps fabrication process, large differential resistance $\left(R_{\mathrm{d}}\right)$, high thermal resistance $\left(R_{\text {thermal }}\right)$, and high threshold current densities $\left(J_{\mathrm{th}}\right)$. Furthermore, small oxide aperture VCSELs tend to produce significant junction heating since the vertical cavity laser diodes modulation bandwidth and output optical power grow in proportion to $\left(I_{\text {bias }}-I_{\text {th }}\right)^{1 / 2}$ and $\left(I_{\text {bias }} I_{\text {th }}\right)$, respectively, where the laser diode must be biased at a current $\left(I_{\text {bias }}\right)$ several times the threshold current $\left(I_{\text {th }}\right)$ in order to reach high performance. Thus, affecting the device reliability.

Conventional VCSELs' structure generally incorporates one or two oxidation layers for electrical and optical confinement. A small and simple change in the metal-organic chemical vapor deposition (MOCVD) recipe results in integrating several oxidation layers within the VCSEL's DBRs, thus 
affecting performance. This paper discusses the effect of utilizing multi-oxide layer structures on VCSEL's performance compared to conventional structure. Related measured data will be presented where applicable.

\section{FABRICATION ISSUES}

One of the advantages of the presented MOL structure is that MOL devices can be fabricated using exactly the same fabrication process and steps sequence used to fabricate conventional structure VCSELs given that DBRs used are similar in terms of thicknesses and composition. VCSELs with MOL structures can be easily realized by modifying only the growth program during the MOCVD process or any other growth technique used.

Typically conventional VCSEL's structure consists of an active region with several quantum wells (QWs) sandwiched between specific number of top and bottom $\mathrm{Al}_{\mathrm{x}} \mathrm{Ga}_{1-\mathrm{x}} \mathrm{As} /$ $\mathrm{Al}_{\mathrm{y}} \mathrm{Ga}_{1-\mathrm{y}} \mathrm{As} \mathrm{DBRs}$, where $\mathrm{x}$ and $\mathrm{y}$ are the $\mathrm{Al}$ mole fractions. To form the oxide aperture, a low-index quarter wavelength layer buried inside the DBRs and adjacent to the optical cavity with high $\mathrm{Al}$ composition $\left(\mathrm{Al}_{0.98} \mathrm{Ga}_{0.02} \mathrm{As}\right)$ is utilized. Fig. 1 illustrates the cross-sectional structure of a fabricated VCSEL

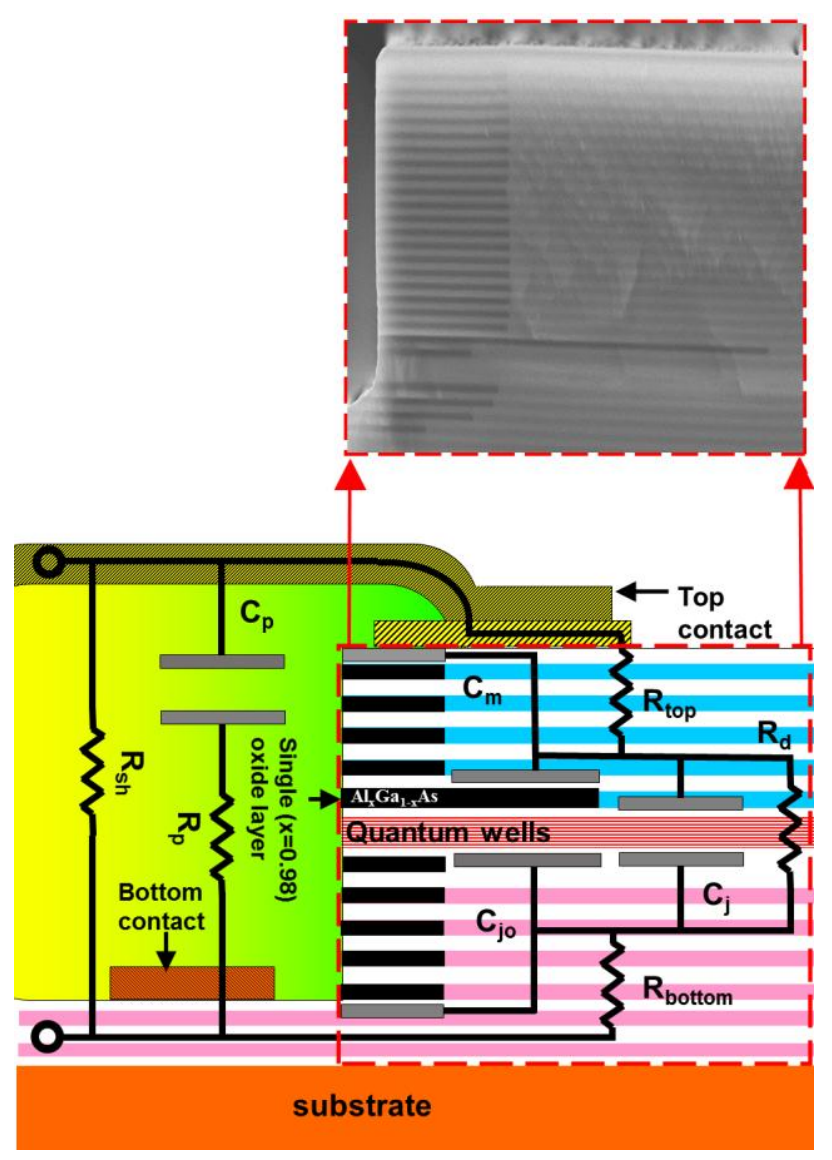

Fig. 1 Cross-sectional structure of a fabricated VCSEL with conventional DBR structure. The inset illustrates an SEM photograph of the VCSEL's mesa showing one oxidation layer extends far inside the structure more than the other oxidized layers. with conventional DBR structure ready for testing. The inset illustrates a scanning electron microscope (SEM) photograph of the VCSEL's mesa showing one oxidation layer extends further inside the structure compared to the other oxidation layers.

The MOL structure is similar to conventional structure except that the $\mathrm{Al}$ content in the layer adjacent to the cavity that is oxidized for optical and current confinement is reduced from $98 \%$ to values ranging from $90 \%$ up to $96 \%$ and increase the $\mathrm{Al}$ content in the $\mathrm{Al}_{\mathrm{y}} \mathrm{Ga}_{1-\mathrm{y}} \mathrm{As}$ layers from $84 \%$ to $90 \%$ up to $96 \%$. Thus, the confinement layer and overlying layers will have the same oxidation rate and hence length as they have the same $\mathrm{Al}$ content. Fig. 2 illustrates the cross-sectional structure of a fabricated VCSEL with MOL DBR structure ready for testing. The inset illustrates an SEM photograph of the VCSEL's mesa showing several oxidation layers extend almost equally inside the structure. The current confinement layer has the same role as the high $\mathrm{Al}$ content (98\%) oxidation layer in conventional oxide VCSELs. Higher-order mode suppression layers are formed above the current confinement layer. While the scattering loss of the fundamental mode can be suppressed to the same level of a conventional VCSEL, these layers give a larger scattering loss and suppress higher-

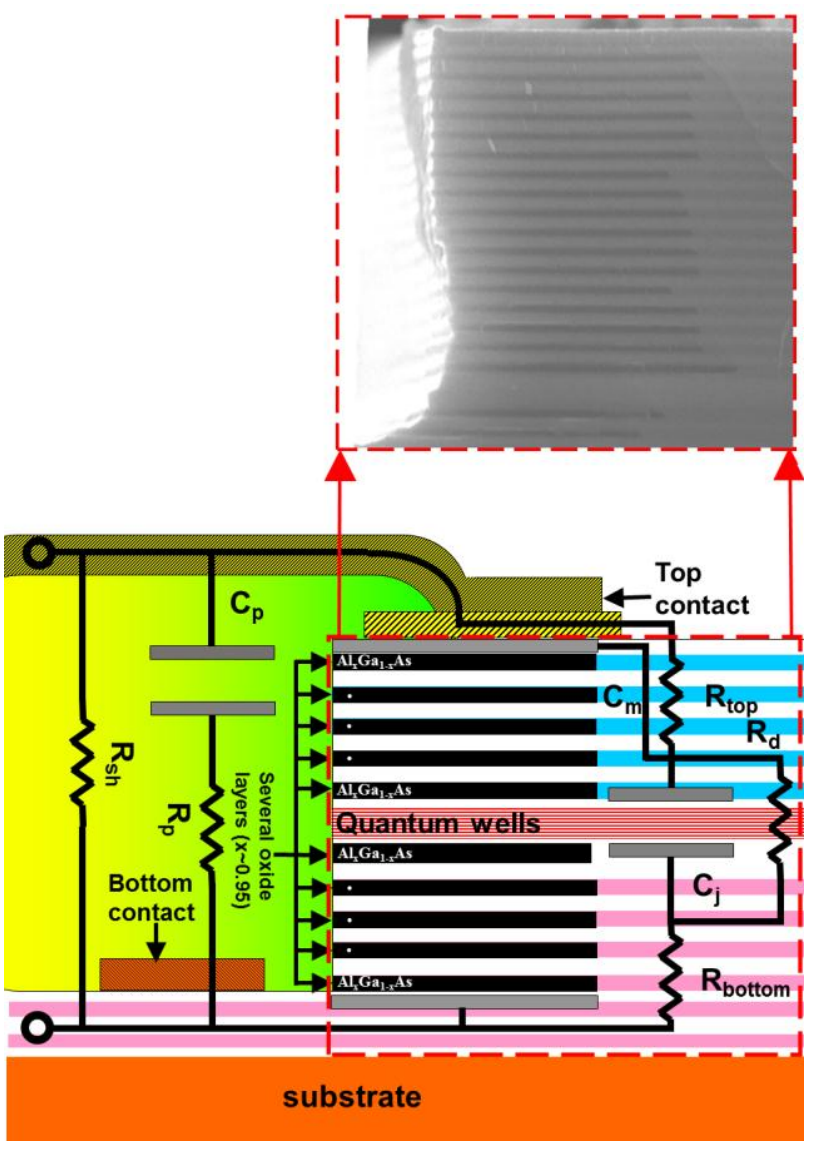

Fig. 2 Cross-sectional structure of a fabricated VCSEL with MOL DBR structure. The inset illustrates an SEM photograph of the VCSEL's mesa showing several oxidation layers extend almost equally inside the structure 
order transverse modes compared to conventional VCSELs. Thus, the mode suppression layers serve as a spatial filter [15], [16].

\section{BANDWIDTH AND PARASITIC CAPACITANCE ISSUES}

Since one of the bandwidth limiting factors that keeps the current devices from reaching their estimated intrinsic response limit is electrical parasitic circuit elements, it is appropriate to present an equivalent circuit model for both conventional and MOL structure VCSELs as shown in Fig. 1 and Fig. 2.

The equivalent circuit consists of the parasitic circuit elements associated with the physical features of the VCSEL's structure that hinder the modulated signal from reaching the VCSEL's junction (i.e. active region) at high modulation frequencies and thus limiting its frequency modulation bandwidth. The presented equivalent circuit model permits the individual investigation of the relative effect of each parasitic parameter on the VCSEL's characteristics. As illustrated in Fig. 1 and Fig. 2 the equivalent circuit model for both conventional and MOL structure VCSELs included the following elements: $R_{\mathrm{p}}$ represents the polyimide dielectric losses, $C_{\mathrm{p}}$ denotes the pad capacitance between the metal pad on the polyimide and the bottom mirror stack, $R_{\mathrm{sh}}$ characterizes the resistance of the dielectric material at low frequency due to pad capacitor leakage current, which is usually on the order of multi-giga ohms, $R_{\text {top }}$ and $R_{\text {bottom }}$ correspond to the upper and lower DBR resistances including the top and bottom contacts resistance, respectively, a parallel combination of a capacitance $C_{\mathrm{j}}$ and a resistance $R_{\mathrm{d}}$ represents the active region junction capacitance and differential resistance, $C_{\mathrm{jo}}$ and $C_{\mathrm{m}}$ represent the mesa region with single oxidized layer while only $C_{\mathrm{m}}$ exists for MOL VCSEL structure as illustrated in Fig. 1 and Fig.2.

As a case study the device structure and some of the results reported in [18] will be utilized. Using conventional DBR structure, $5 \mu \mathrm{m}$ height top-emitting, high-speed, $850 \mathrm{~nm}$ VCSELs were fabricated and characterized. Devices with 10 $\mu \mathrm{m}$ oxide aperture and $31 \mu \mathrm{m}$ mesa diameter exhibited a maximum measured $-3 \mathrm{~dB}$ modulation bandwidth of $15.3 \mathrm{GHz}$ and an estimated parasitic limited $-3 \mathrm{~dB}$ modulation bandwidth of $21.5 \mathrm{GHz}$ [18]. The parasitic capacitances $C_{\mathrm{p}}, C_{\mathrm{j}}, C_{\mathrm{jo}}$, and $C_{\mathrm{m}}$ shown in Fig. 1 were estimated based on geometrical considerations and materials characteristics. Values for the parasitic resistances $R_{\mathrm{p}}, R_{\mathrm{top}}+R_{\text {bottom }}$, and $R_{\mathrm{d}}$ were extracted based on measured data

To investigate the MOL structure effect on the VCSEL's modulation performance, the VCSEL equivalent circuit model driven from a source with an output resistance of $R_{\text {source }}=50 \Omega$ as illustrated in Fig. 3 was simulated using PSpice. As for the circuit model elements, values used were $C_{\mathrm{p}}=69 \mathrm{fF}, C_{\mathrm{j}}=53 \mathrm{fF}$, $C_{\mathrm{m}}=6 \mathrm{fF}, R_{\mathrm{p}}=10 \Omega, R_{\text {top }}+R_{\text {bottom }}=42 \Omega$, and $R_{\mathrm{d}}=50 \Omega$. During simulation, all circuit elements (capacitances and resistances) values were fixed except $C_{\mathrm{jo}}$ value was varied. This variation corresponds to change in oxidation layers number. Assuming that $C_{\mathrm{jo}}$ can be treated as a parallel plate capacitor, then its capacitance can be estimated using $C_{j o}=\varepsilon_{o} \varepsilon_{r} A_{j o} / d$. Where $\varepsilon_{\mathrm{o}}$ is the free space permittivity $\left(8.85 \times 10^{-14} \mathrm{~F} / \mathrm{cm}\right), A_{\mathrm{jo}}$ is the area, $d$ is the thickness, $\varepsilon_{\mathrm{r}}$ is the Aluminum oxide $\left(\mathrm{Al}_{2} \mathrm{O}_{3}\right)$ dielectric constant at low frequency ( 9). Thus, as the number of oxidation layers increase, $d$ will increase and as a result $C_{\text {jo }}$ values will decrease and vice versa. Fig.4 exhibits the simulated maximum possible small signal modulation response for VCSELs with conventional and MOL structures. As shown in Fig.4, results indicated that about $68 \%$ improvement in the maximum $-3 \mathrm{~dB}$ modulation frequency, where conventional VCSELs exhibited an $f_{-3 \mathrm{~dB} \text { max }}$ value of $22 \mathrm{GHz}$ while MOL VCSELs exhibited an $f_{-3 \mathrm{~dB} \text { max }}$ value of $37 \mathrm{GHz}$. Simulated small signal modulation response as a function of $C_{\mathrm{jo}}$ (i.e. various $C_{\text {jo }}$ values corresponds to different number of oxide layers) is illustrated in Fig. 5. Where results clearly indicate that reducing $C_{\mathrm{jo}}$ by increasing the number of oxidation layers will increase the $f_{-3 \mathrm{~dB}}$.

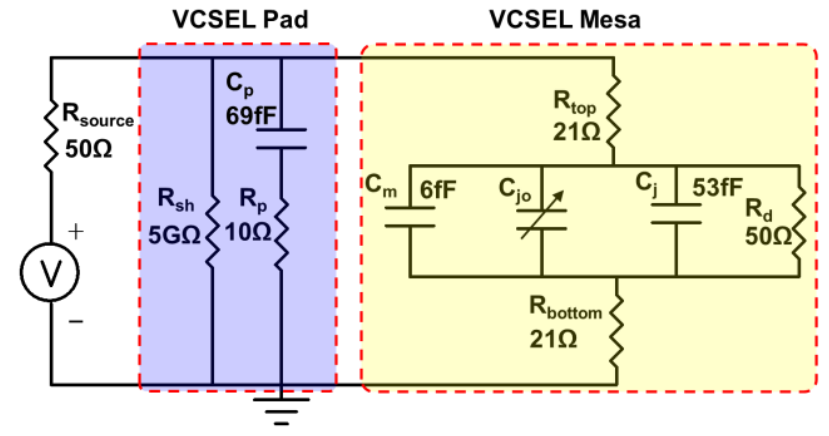

Fig.3 Simplified VCSEL equivalent circuit model driven from a source with an output resistance of $R_{\text {source }}=50 \Omega$.

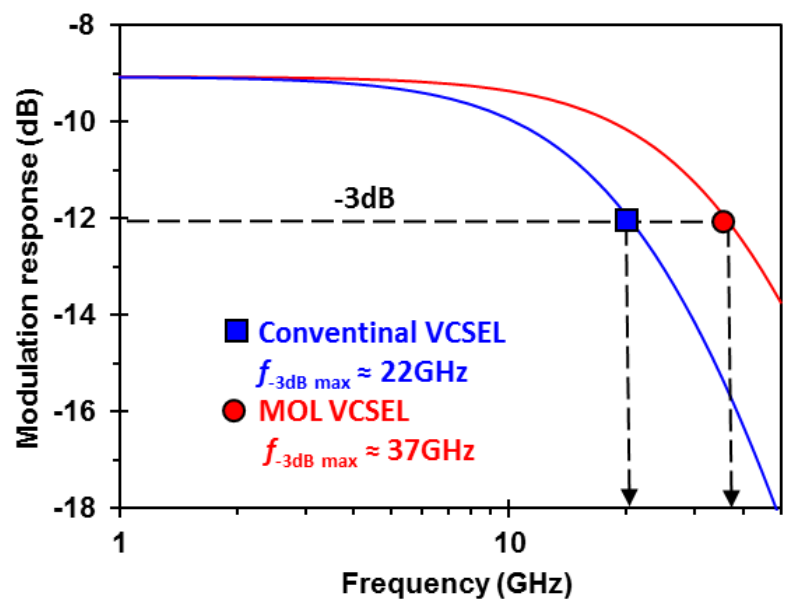

Fig.4 Simulated maximum possible small signal modulation response for VCSELs with conventional and MOL structures

\section{Single-Mode AND Thermal Issues}

As mentioned earlier, multi-mode operation can also limit the VCSEL's performance and thus limits the utilization of VCSELs in several potential applications. The presented MOL structure not only reduces the parasitic capacitance and thus improves the $f_{-3 \mathrm{~dB} \max }$, but also helps in suppressing higherorder mode. While the scattering loss of a fundamental mode 
can be suppressed to the same level of a conventional VCSEL, these layers (oxidation layers) give a larger scattering loss and suppress higher-order transverse modes compared to conventional VCSELs. Thus, the MOL structure serves as a spatial filter. In previously reported results [15], the inclusion of 17 oxidation layers in $8 \mu \mathrm{m}$ oxide aperture diameter VCSELs helped maintain single-mode operation with a side mode suppression ratio (SMSR) of more than $32 \mathrm{~dB}$ at driving bias currents up to 20 times threshold current.

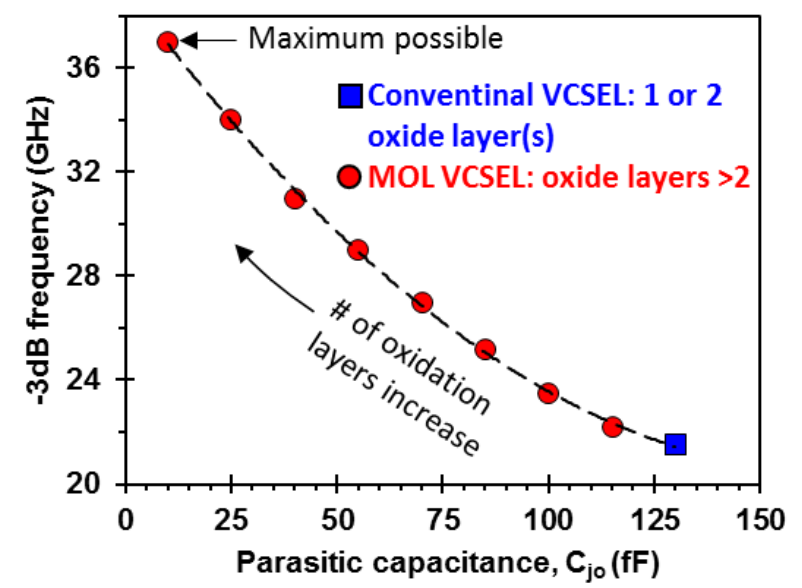

Fig. 5 Simulated small signal modulation response as a function of $C_{\mathrm{jo}}$ (i.e. different number of oxide layers)

Using the typical value for optical mode shift $\partial \lambda / \partial \mathrm{T} \approx$ $0.07 \mathrm{~nm} /{ }^{\circ} \mathrm{C}$ in GaAs-based VCSELs, the thermal resistance $\left(R_{\mathrm{th}}\right)$ is typically determined by measuring the wavelength $(\lambda)$ dependence of the laser spectra as a function of the electrical input power $\left(P_{\text {in }}\right)$. MOL VCSELs with $8 \mu \mathrm{m}$ active diameter reported in [15] exhibited a measured $R_{\text {th }}$ of $\sim 6^{\circ} \mathrm{C} / \mathrm{mW}$. To put this result into context and compare with conventional VCSELs, the analytical estimation $R_{\text {thermal }}=1 / 2 \xi d$ was used to evaluate the VCSEL's structure thermal conductivity $(\xi)$, where $d$ is the VCSEL oxide aperture diameter. Calculations resulted in a very low thermal conductivity value of 0.084 $\mathrm{W} / \mathrm{cm}-\mathrm{K}$, which is very low compared to the thermal conductivity of $\mathrm{GaAs}(\xi=0.45 \mathrm{~W} / \mathrm{cm} . \mathrm{K}), \mathrm{Al}_{0.1} \mathrm{Ga}_{0.9} \mathrm{As}(\xi=0.2$ $\mathrm{W} / \mathrm{cm} . \mathrm{K}), \mathrm{Al}_{0.9} \mathrm{Ga}_{0.1} \mathrm{As}(\xi=0.25 \mathrm{~W} / \mathrm{cm} . \mathrm{K})$, and AlAs $(\xi=0.9$ $\mathrm{W} / \mathrm{cm} . \mathrm{K})$. It is believed that this degradation in the estimated thermal conductivity is due to the very low oxidation layers thermal conductivity value of $0.007 \mathrm{~W} / \mathrm{cm} . \mathrm{K}$ and poor heat sinking as a result of using polyimide for mesa wrapping, which has a very low thermal conductivity of $0.002 \mathrm{~W} / \mathrm{cm} .{ }^{\circ} \mathrm{C}$ [2]. It is believed that with improved heat sinking such as using plated Copper [2] and optimized multiple oxide layer [15], [16] structure should increase the MOL structure thermal conductivity and thus improve the device's reliability

\section{V.CONCLUSION}

The MOL structure expected effect on VCSEL's performance was presented. PSpice simulation results indicated $\sim 68 \%$ improvement in $f_{-3 \mathrm{~dB}}$ max, where conventional and MOL VCSELs exhibited $f_{-3 \mathrm{~dB}} \max$ values of $22 \mathrm{GHz}$ and
$37 \mathrm{GHz}$, respectively. Also simulations indicated that reducing $C_{\mathrm{jo}}$ by increasing the number of oxidation layers will increase the $f_{-3 \mathrm{~dB}}$. Furthermore, MOL VCSELs maintain single mode operation with a SMSR > $32 \mathrm{~dB}$ at high bias currents. On the other hand, MOL structure demonstrated a degraded performance in terms reduced $\xi$ of $0.084 \mathrm{~W} / \mathrm{cm}-\mathrm{K}$ compared to GaAs and AlAs. It is believed that with improved heat sinking such as using plated metal $(\mathrm{Cu}$ or $\mathrm{Au})$ and optimized multiple oxide layer structure the MOL structure should increase $\xi$ and thus improve the device's reliability.

\section{REFERENCES}

[1] L. Ya. Karachinsky, S. A. Blokhin, I. I. Novikov, N. A. Maleev, A. G. Kuzmenkov, M. A. Bobrov, J. A. Lott, N. N. Ledentsov, V. A. Shchukin, J-R. Kropp and D. Bimberg, "Reliability performance of 25 Gbit s-1 $850 \mathrm{~nm}$ vertical-cavity surface-emitting Lasers," Semicond. Sci. Technol. vol.28, pp. 065010(8pp), 2013.

[2] A. N. AL-Omari, A. Ababneh, and K. L. Lear, "High-Speed InvertedPolarity Oxide-Confined Copper-Plated 850-nm Vertical-Cavity Lasers," IEEE J. Sel. Top. Quantum Electron., Vol.21, No.6, 1701408, November/December 2015.

[3] P. Moser, J. A. Lott, and D. Bimberg, "Energy Efficiency of Directly Modulated Oxide-Confined High Bit Rate 850-nm VCSELs for Optical Interconnects" IEEE J. Sel. Top. Quantum Electron., vol. 19, no. 4, JULY/AUGUST 2013.

[4] M. Gruber, "Multichip module with planar-integrated free-space optical vector-matrix-type interconnects," Appl. Opt., vol. 43, pp. 463-470, Jan. 2004.

http://dx.doi.org/10.1364/AO.43.000463

[5] A.K. Dutta, H. Kosaka, K. Kurihara, Y. Sugimasa, and K. Kasahara, "High-speed VCSEL of modulation bandwidth over $7.0 \mathrm{GHz}$ and its application to 100 m PCF datalink," IEEE J. Lightwave Technol., 1998, 16 , pp. 870-875. http://dx.doi.org/10.1109/50.669024

[6] D. Kuchta, C. Schow, A. Rylyakov, J. Proesel, F. Doany, C. Baks, B. Hamel-Bissell, C. Kocot, L. Graham, R. Johnson, G. Landry, E. Shaw, A. MacInnes, and J. Tatum, "A $56.1 \mathrm{~Gb} / \mathrm{s}$ NRZ Modulated $850 \mathrm{~nm}$ VCSEL-Based Optical Link," Optical Fiber Communication Conference, Optical Society of America, Anaheim, CA, USA, p. OW1B.5, March 2013.

http://dx.doi.org/10.1364/ofc.2013.ow1b.5

[7] A. N. AL-Omari and K. L. Lear, "Polyimide-Planarized Vertical-Cavity Surface Emitting Lasers with 17.0 GHz Bandwidth," IEEE Photon. Technol. Lett., vol. 16, no. 4, pp. 969-971, April 2004. http://dx.doi.org/10.1109/LPT.2004.824622

[8] P. Westbergh, R. Safaisini, E. Haglund, J. S. Gustavsson, A. Larsson, M. Geen, R. Lawrence, and A. Joel," High-Speed Oxide Confined 850$\mathrm{nm}$ VCSELs Operating Error-Free at $40 \mathrm{~Gb} / \mathrm{s}$ up to $85{ }^{\circ} \mathrm{C}$," IEEE Photon. Technol. Lett., vol. 25, no. 8, pp.768-771, April 2013. http://dx.doi.org/10.1109/LPT.2013.2250946

[9] A. Larsson, P. Westbergh, J. Gustavsson, Å. Haglund and B. Kögel, "High-speed VCSELs for short reach communication," Semicond. Sci. Technol. vol.26, pp.014017(5pp), 2011

[10] J. W. Shi, J. C. Yan, J. M. Wun, J. Chen, and Y. J. Yang, "Oxide-Relief and Zn-Diffusion 850-nm Vertical-Cavity Surface-Emitting Lasers With Extremely Low Energy-to-Data-Rate Ratios for $40 \mathrm{Gbit} / \mathrm{s}$ Operations." IEEE J. Sel. Top. Quantum Electron., vol.19, no.2 pp. 79002087900208, March/April 2013. http://dx.doi.org/10.1109/JSTQE.2012.2210863

[11] A. N. Al-Omari, G. P. Carey, S. Hallstein, J. P. Watson, G. Dang, and K. L. Lear, "Low thermal resistance high-speed top-emitting 980-nm VCSELs," IEEE Photon. Technol. Lett., vol. 18, no. 11, pp. 1225-1227, May-Jun. 2006.

http://dx.doi.org/10.1109/LPT.2006.875059

[12] R. Safaisini, J. R. Joseph, D. Louderback, X. Jin, A. N. AL-Omari, and Kevin L. Lear,“ Temperature Dependence of 980-nm Oxide-Confined VCSEL Dynamics," IEEE Photon. Technol. Lett. vol. 20, no.14, pp.1273-1275, July 2008. 
http://dx.doi.org/10.1109/LPT.2008.926564

[13] A. N. AL-Omari and K. L. Lear, "Fabrication and performance of bottom-emitting flip-chip bonded $980 \mathrm{~nm}$ vertical-cavity lasers with copper- and indium-plated heat-sinks," Semicond. Sci. Technol., vol. 26, no.12, pp. 125020 (9pp), November 2011.

[14] K. L. Lear and A. N. AL-Omari, "Progress and issues for high-speed vertical cavity surface emitting lasers", Proc. Of SPIE - The International Society for Optical Engineering, Vertical-Cavity SurfaceEmitting Lasers XI Conf., 6484, San Jose, CA, USA, pp.145-156, March 2007.

[15] A.N. Al-Omari and K.L. Lear, "High-speed $980 \mathrm{~nm}$ vertical cavity surface emitting lasers with a multi-oxide layer structure for singlemode operation ", IET Optoelectron., vol.5, No.2, pp. 57-61, April 2011.

http://dx.doi.org/10.1049/iet-opt.2009.0059

[16] N. Nishiyama, M. Arai, S. Shinada, K. Suzuki, F. Koyama, and K. Iga, "Multi-oxide layer structure for single-mode operation in vertical-cavity surface emitting lasers," IEEE Photon. Technol. Lett., 2000, 12, pp. 606-608.

http://dx.doi.org/10.1109/68.849058

[17] A. N. AL-Omari, M. S. Alias, A. Ababneh and K. L. Lear, "Improved performance of top-emitting oxide-confined polyimide-planarized 980nm VCSELs with copper-plated heat sinks," J. Phys. D: Appl. Phys. vol. 45, no. 50, pp. 505101 (8pp) Dec. 2012.

[18] A. N. AL-Omari, I. K. AL-Kofahi, and K. L. Lear, "Fabrication, performance and parasitic parameter extraction of $850 \mathrm{~nm}$ high-speed vertical-cavity lasers," Semicond. Sci. Technol., vol. 24, no.9, pp. 095024 (8pp), September 2009. 